\title{
DIGITALCOMMONS $@$ WAYNESTATE-

Peritoneal dialysis vs. hemodialysis: The choice of modality of dialysis may drastically affect the quality of life of patients initiating dialysis

Deepa Daryani

Wayne State University, gm2692@wayne.edu

Follow this and additional works at: https://digitalcommons.wayne.edu/crp

Part of the Internal Medicine Commons, Medical Education Commons, and the Nephrology Commons

\section{Recommended Citation}

DARYANI D. Peritoneal dialysis vs. hemodialysis: The choice of modality of dialysis may drastically affect the quality of life of patients initiating dialysis. Clin. Res. Prac. Oct 13 2021;7(2):eP2579. https://doi.org/ $10.22237 / \mathrm{crp} / 1625097840$

This Clinical Decision Report is brought to you for free and open access by the Open Access Journals at DigitalCommons@WayneState. It has been accepted for inclusion in Clinical Research in Practice: The Journal of Team Hippocrates by an authorized editor of DigitalCommons@WayneState. 


\section{Peritoneal dialysis vs. hemodialysis: The choice of modality of dialysis may drastically affect the quality of life of patients initiating dialysis}

DEEPA DARYANI, Wayne State University School of Medicine, gm2692@wayne.edu

\section{ABSTRACT A clinical decision report using}

Jung HY, Jeon Y, Park Y, et al. Better Quality of Life of Peritoneal Dialysis compared to Hemodialysis over a Two-year Period after Dialysis Initiation. Sci Rep. Jul 16 2019;9(1):10266. https://doi.org/10.1038/s41598-019-46744-1

for a young patient initiating dialysis.

Keywords: $\quad$ peritoneal dialysis, hemodialysis, quality of life

\section{Clinical-Social Context}

William Smith [pseudonym] is a 46 year old African American male with a past medical history significant for hypertension and Stage 5 chronic kidney disease (CKD) who presented for shortness of breath with associated bilateral lower extremity swelling and cough. The patient stated that he had a milder form of these symptoms for the last 4 months, which was when he was initially informed of his diagnosis of stage 5 CKD, that was most likely due to his uncontrolled hypertension. At that time, the patient was told his best option for treatment would be to initiate either peritoneal dialysis or hemodialysis. The patient was given proper education about both dialysis modalities and their necessity for treatment, however the patient refused initiation of dialysis. As a separated middle-aged male who lived alone and was in the process of rekindling his relationship with his wife, the patient felt he was too young and too occupied in personal aspects of his life to be debilitated by the rigorous dialysis regimen necessary for his condition. Additionally, though the patient had his own car for transportation, he already worked 6 days a week in a physically demanding job to make enough money to be financially secure. Therefore, from an economic standpoint, he did not think he could afford the increased transportation costs and the necessary time off work he would have to take to undergo dialysis. Instead, the patient had opted to see transplant nephrology in hopes of getting a kidney transplant, but currently was without a donor. Therefore, since his diagnosis the patient had not been receiving any treatment for his stage 5 CKD and has had progressive worsening of his symptoms.

On presentation to the ED, the patient was hypertensive, severely short of breath, and visibly distressed on two liters of oxygen by nasal cannula. A workup was done, which pointed to worsening kidney function. The patient was given symptomatic treatment and was transferred to the Nephrology floor. At that point, the patient was offered a treatment option of emergent hemodialysis. However, the patient was uncomfortable with starting

DEEPA DARYANI is a $4^{\text {th }}$ year medical student at the Wayne State University School of Medicine. 
DARYANI D. Peritoneal dialysis vs. hemodialysis: The choice of modality of dialysis may drastically affect the quality of life of patients initiating dialysis. Clin. Res. Prac. Oct 13 2021;7(2):eP2579. https://doi.org/10.22237/crp/1625097840
Clinical Research in Practice The Journal of Team Hippocrates

VOL 7 ISS 2 / eP2579 / OCTOBER 13, 2021 https://doi.org/10.22237/crp/1625097840

hemodialysis so urgently. He was treated symptomatically with a diuretic challenge of Bumex $6 \mathrm{mg}$ IV and metolazone, and his options were discussed amongst the medical team.

After a few hours of worsening symptoms of orthopnea, the patient accepted emergent port placement and hemodialysis. He also accepted the commencement of a dialysis regimen, but re-iterated his confusion about which modality would fare well with his lifestyle.

\title{
Clinical Question
}

Which renal replacement therapy modality, hemodialysis or peritoneal dialysis, is better for the treatment of end stage renal disease for patients initiating dialysis, using quality of life as a major deciding factor?

\section{Research Article}

Jung HY, Jeon Y, Park Y, et al. Better Quality of Life of Peritoneal Dialysis compared to Hemodialysis over a Two-year Period after Dialysis Initiation. Sci Rep. Jul 16 2019;9(1):10266. https://doi.org/10.1038/s41598-019-46744-1 ${ }^{1}$

\section{Description of Related Literature}

\author{
A search of PubMed was done using following fields:
}

(peritoneal dialysis) AND (hemodialysis) AND (quality of life)

This initial search yielded a total of 1,357 results. A filter for "randomized controlled studies" and "clinical trials" was applied which eliminated 1,306 articles from the search. The remaining 51 articles were examined. Of these, 45 published articles were removed because they were not relevant to the clinical question. The remaining 6 studies chosen specifically addressed the direct comparison between hemodialysis and peritoneal dialysis and their effect on the quality of life of end stage renal disease patients. To ensure no relevant articles were missed, an additional search was done using Google Scholar. The title of the paper eventually chosen for critical appraisal was entered into Google Scholar and "Related Articles" was selected, which resulted in 101 articles. However, these articles also did not directly address the aspect of quality of life in dialysis modalities, so no additional studies were included. The 6 relevant studies were assessed.

Czyżewski et al. is a cross-sectional study that used a total of 120 patients that were differentiated into 3 groups depending on renal replacement therapy (RRT) method: 30 peritoneal dialysis (PD) patients, 40 hemodialysis (HD) patients, and 47 post-kidney transplantation (KTx) patients. $\stackrel{2}{-}$ The following research tools were used to assess for quality of life: Medical Outcomes Study 36 - the Short Form, Kidney Disease Quality of Life Short Form, and disease history. In dialysis patients, health related quality of life (HRQOL) was measured once, while for post-KTx patient, HRQOL was measured in the 3rd and 12th month after the operation. The results of the study showed that HRQOL of end stage renal disease (ESRD) patients did differ depending on the RRT method: top values were shown by post-KTx patients, lower by PD patients, and the bottom ones by HD patients. This study was rejected because it studied an additional group, post-kidney transplant patients, in the assessment of quality of life with renal replacement therapy. Unfortunately, this RRT is not an option for our patient, so this study does not apply in this clinical context.

Surendra et al. performed a cost utility analysis study on 64 peritoneal dialysis patients and 77 hemodialysis patients from five large Ministry of Health centers in Malaysia over a one year period ranging from October 1, 2016 to September 30, 2017. - The outcomes measured were mean costs per patient per year and cost per quality adjusted life year. The resources that were used included patient responses to the EQ-5D questionnaire, which measured 5 health dimensions; mobility, self-care, usual activities, pain/discomfort, and anxiety/depression, and the Kaplan-Meier product-limit survivor function approach, which estimated the mean survival rates (in life years) for HD and PD patients. The findings suggest both hemodialysis and peritoneal dialysis are fiscally feasible, however increasing PD as initial dialysis modality would be more cost-effective. This study was rejected because it primarily focused on cost effectiveness of different forms of dialysis, which is only one parameter in the quality of life that our patient is focused on. Due to its limited focus on economics, it was rejected. 
DARYANI D. Peritoneal dialysis vs. hemodialysis: The choice of modality of dialysis may drastically affect the quality of life of patients initiating dialysis. Clin. Res. Prac. Oct 13 2021;7(2):eP2579.

https://doi.org/10.22237/crp/1625097840
Clinical Research in Practice The Journal of Team Hippocrates

VOL 7 ISS 2 / eP2579 / OCTOBER 13, 2021 https://doi.org/10.22237/crp/1625097840

Majkowicz M et al performed a cross-sectional study in the period from April 1995 to March 1996 with 65 HD patients and 22 PD patients who were being dialyzed at the Department of Nephrology at the Medical University of Gdansk, and 76 healthy volunteers who served as controls. ${ }^{4}$ The EORTC Quality of life Questionnaire-QLQ-C30 was used to assess quality of life. The results showed that the quality of life of PD patients was similar to the control patients, with minimal differences in personal and social functioning. Hemodialysis patients contrasted unfavorably with controls on the majority of examined scales on quality of life. Though this was a strong study, it was ultimately excluded due to a lack of detail in the methods section, which provided vague insight into how the study was conducted.

The study done by Cirillo L et al was a monocentric cross-sectional study, where 145 patients with at least 3 months of dialysis were screened by Patient Health Questionnaire-9 (PHQ-9), a self-administered depression-screening questionnaire. .5 Depressive symptoms were found in 69 patients (46\%), affecting $36 \%$ of patients on PD versus $52 \%$ of patients on HD. Moreover, the PD patients had significantly less functional impairment derived from depressive symptoms than the HD patients. Though the study used a simple and reliable tool in its analysis, the outcome measured is too narrow to be applied to this patient.

Korevaar JC et al completed a randomized controlled trial with 38 patients who were using dialysis as their first renal replacement therapy option from January 1997 to August 2000. $\underline{6}$ The patients were randomized into two groups: 18 patients to hemodialysis and 20 to peritoneal dialysis. Both groups were asked to complete a EuroQol questionnaire, a standardized generic description of health status that is comprised of five dimensions: mobility, self-care, usual activities, pain/discomfort, and anxiety/depression, at the start of dialysis, 3 and 6 months later, and every 6 months afterward. 13 of the 18 patients in the hemodialysis group and 15 of the 20 randomized to the peritoneal dialysis group completed the EuroQol questionnaire and were able to undergo quality adjusted life year (QALY) analysis. The QALY score for hemodialysis patients was 59.2 (11.8) and 54.4 (21.9) for peritoneal dialysis patients, a difference of $4.8(-9.1$ to 18.8 ) (P value $=0.47)$. Ultimately, the results showed that there was no statistically significant difference in mean QALY score observed between patients starting hemodialysis vs peritoneal dialysis. Though the study provides a general overview of the relationship between quality of life and the mode of dialysis, the specific factors of quality of life for the patient in question, such as finances and personal relationships, were not clearly identified in this study. Additionally, due to the low number of participants and the corresponding low power of the study, this study was not deemed strong enough to answer the clinical question.

The only suitable prospective cohort study selected for critical appraisal, entitled "Better Quality of Life of Peritoneal Dialysis compared to Hemodialysis over a Two-year Period after Dialysis Initiation.", studies the clinical question of interest and also identifies specific parameters of quality of life relevant to the patient as a primary endpoint. . $^{1}$

Though a randomized controlled trial would typically be considered an ideal study with the most strength and validity, due to the minimal number of articles available on this subject, cohort studies were considered. This particular study used the largest sample of patients, followed patients over the longest period of time, and was able to adequately evaluate changes in specific parameters of quality of life relative to other studies, and therefore was deemed to be the strongest study available. Based on Strength of Recommendation Taxonomy (SORT) criteria, the above body of evidence would be a grade B, given that there are limited studies examining the relationship between dialysis modalities and quality of life. ${ }^{7}$

\section{Critical Appraisal}

This study by Jung et al is a prospective nationwide cohort study trial from 31 different hospital centers in South Korea that recruited 2199 patients, ages 19 or older who were using dialysis as their first renal replacement therapy option. The goal of the study was to compare patients' reported feelings on quality of life over the span of 24 months while undergoing either peritoneal or hemodialysis, and to expand the strength of evidence by using a prolonged prospective cohort study rather than a short observational study, which has been the norm for analysis of quality of life thus far. This selected study meets the criteria for level 2 quality of evidence based on the SORT criteria. ${ }^{7}$

The patients were distinguished into two groups based on their initial dialysis modality of choice: 1,581 patients to hemodialysis and 618 to peritoneal dialysis. Both groups underwent typical dialysis protocol and were asked to complete the KDQOL-SFTM 1.3 questionnaire, a standardized generic description of health status that consists of 80 items. The 80 items were used to calculate 3 different scores -- the results of the ESRD-targeted items were aggregated into kidney disease composite score (KDCS), the results of 
DARYANI D. Peritoneal dialysis vs. hemodialysis: The choice of modality of dialysis may drastically affect the quality of life of patients initiating dialysis. Clin. Res. Prac. Oct 13 2021;7(2):eP2579. https://doi.org/10.22237/crp/1625097840
Clinical Research in Practice The Journal of Team Hippocrates

VOL 7 ISS 2 / eP2579 / OCTOBER 13, 2021 https://doi.org/10.22237/crp/1625097840

the physical health items were classified into physical composite score (PCS), and lastly items related to emotional wellbeing were calculated into a mental composite score (MCS). This questionnaire, along with general sociodemographic information, and clinical and biochemical data were obtained 3, 12, and 24 months after the start of dialysis. The mean age of the HD group was $56.5+/-13.5$ and the mean age of the PD group was $51.6+/-12.8$. The mean residual renal function was $10.7 \mathrm{~mL} / \mathrm{min} / 1.73 \mathrm{~m}{ }^{2} \mathrm{for}$ the hemodialysis group and $11.1 \mathrm{~mL} / \mathrm{min} / 1.73 \mathrm{~m}^{2}$ for the peritoneal dialysis group at the first measured time period of 3 months. The baseline characteristics of both groups were similar to the medical profile of our patient, however the mean age was slightly higher. Though the study did not comment on the ethnic demographics of their study population, they used reference values for an East Asian population. This could raise concerns of clinical correlation due to our patient being a slightly younger African American male from the United States.

The study protocol did not include randomization to separate patients. This provided a situation in which there could be a concern for confounding bias. However, this was accounted for as the results of the KDQOL-SFTM 1.3 questionnaire were adjusted for age, sex, educational level, employment status, marital status, hemoglobin, albumin, and total cholesterol levels. The authors used multivariate logistic regression analysis to investigate the association between questionnaire scores and background variables. As there was no significant difference in the factors observed between the two groups, there was low concern for indication bias.

Results were presented as mean changes in questionnaire scores between 3 to 24 months. Patients on HD experienced significantly worsened HRQOL in the three ESRD domains of sexual function $(-9.6, P=0.005)$, sleep $(-2.7, P=0.04)$, and patient satisfaction $(-3.5$, $P=0.04)$ but improved HRQOL in one PCS domain, role-physical $(10.4, P=0.002)$. Patients on PD underwent significantly worsened HRQOL in the two ESRD domains of burden of kidney disease $(-5.3, P=0.009)$ and work status $(-6.8, P=0.03)$, in one PCS domain, general health $(-3.8, P=0.02)$, and in two MCS domains, emotional wellbeing $(-3.4, P=0.02)$ and energy/fatigue $(-3.1, P=0.04)$. However, no significant differences in changes in overall HRQOL over time were observed between dialysis modalities. As there was no significant difference between the two interventions, effect size was negligible.

Though quite a unique study, there were some apparent weaknesses. Firstly, the biggest limitation is in the design. There was a lack of randomization, though the baseline characteristics were continuously adjusted for. The methods section of this study was quite vague and did not provide adequate detail on how patients were chosen, leading us to believe there was high potential for selection bias. It is unclear whether the patients were already given information about the varying dialysis modalities which could have altered their initial dialysis modality choice and their perceptions of HRQOL, thereby having a massive impact on the primary measure of the study results. Furthermore, there is no information on measured pre-dialysis quality of life, which might have yielded more useful results. There is also no information on baseline characteristics at the start of the study, as first measurements were recorded at the 3-month mark. The lack of baseline inclusion criteria makes it difficult to ascertain how clinically relevant this study is to our patient. Additionally, there was a notable amount of attrition bias in the study, seen in decrease of the response rate of the questionnaire over time, more so in the HD group than in the PD group. Specifically, 1581 patients were initially in the HD group and 618 in the PD group. At 3 months only $42.2 \%$ of patients on HD and $54.9 \%$ on PD completed the questionnaire. At 12 months, $20.6 \%$ of patients on HD and $31.7 \%$ on PD completed the questionnaire. At 24 months, $10.8 \%$ of patient on HD and $19.2 \%$ on PD completed the questionnaire. The reason for non-response was not adequately described in the study. Also, from the patients that started with HD, a total of 193 patients had died, and from the patients that started with PD, a total of 35 patient died. As patients passed away or become non-responders, they were dropped from the study. Lastly, though the follow up period of 2 years was longer than other studies, it was not long enough to see the actual effects of long-term kidney damage and the deterioration of renal function. Therefore, this study was unable to capture enough data to demonstrate the association of gradual deterioration of renal function and quality of life in dialysis patients.

Nevertheless, the study has strengths in that it provided a unique method in evaluating the quality of life of patients amongst two modalities of dialysis, with a longer duration of follow-up compared with previous studies. Additionally, though HRQOL was generalized utilizing the questionnaire scores, specific aspects of quality of life were trended via the KDCS, PCS, and MCS scores which allowed for more precise details on which aspects of quality of life were affected. This allowed the study to provide more conclusive data relative to prior cohort studies. Despite its limitations, the study, while showing no significant difference in overall HRQOL, may be useful to patients considering specific outcomes of peritoneal vs hemodialysis. The study is important in clarifying that both modalities have varying effects on different aspects of general quality of life. 
DARYANI D. Peritoneal dialysis vs. hemodialysis: The choice of modality of dialysis may drastically affect the quality of life of patients initiating dialysis. Clin. Res. Prac. Oct 13 2021;7(2):eP2579. https://doi.org/10.22237/crp/1625097840
Clinical Research in Practice The Journal of Team Hippocrates

VOL 7 ISS 2 / eP2579 / OCTOBER 13, 2021 https://doi.org/10.22237/crp/1625097840

The study could be reproduced feasibly, given the necessary amount of time to gather and follow patients starting renal replacement therapy. Overall, the study gave a unique approach to identifying the differences in quality of life amongst dialysis patients, however there is still much research to be done in this field as results seem to be varying between clinical articles. The funding source had no role in the collection, analysis, or interpretation of the data or in the decision to submit the manuscript for publication.

\section{Clinical Application}

This study can be applied to Mr. Smith's case as he meets inclusion criteria since he is a patient greater than 19 years old who is initiating dialysis for the first time. However, as the study showed equivalence between hemodialysis and peritoneal dialysis in terms of overall HRQOL scores, the decision to start with one modality versus the other is more dependent on other social considerations specific to the patient. Mr. Smith was mostly concerned about the timing and cost of the dialysis routine, and the associated effect on mental health and his relationship, which could prevent him from meeting his wife and going to his job. Concerning cost, studies show that PD costs are significantly lower than HD costs. In regard to personal relationships, there was less effect on sexual function and overall patient satisfaction with PD relative to HD. Also, unlike patients on PD, patients undergoing HD must go to dialysis centers two to three times a week for four hours per session. This requirement can negatively affect both personal lives and occupational achievement. This study shows that patients on PD were bothered less by the burden of ESRD, symptoms, and pain, and were able to continue their jobs more compared with those on HD. 1

A discussion was had with Mr. Smith addressing his concerns about adjusting to the initiation of dialysis and the possible effect on his quality of life. Although he was initially apprehensive, we explained that due to the other concerns in his life, peritoneal dialysis would be the best option for him and he agreed. We spent time explaining how to use the machine, scheduled him with an outpatient nephrology follow up in order to exchange his current HD site with a peritoneal site, and also planned for a caregiver to assist him at home. We had a family meeting that including his wife who stated she would come by to assist the patient with his new needs. This conversation eased the patient's stress about his new peritoneal dialysis and made him amendable to his plan upon discharge.

\section{New Knowledge Related to Clinical Decision Science}

The initiation of dialysis, regardless of which modality, can be a major burden on finances, social relationships, and mental and physical health. This was the main concern for Mr. Smith who was initially hesitant to begin treatment because of the difficult choice he had to make in between dialysis options. The decision between hemodialysis and peritoneal dialysis was discussed with special attention to the patients concerns such as time with family and cost of treatment, amongst other quality of life issues. In this case, a critical review of the literature revealed that overall health related quality of life was equivalent between the two modalities, with significant differences noted in specific parameters of life including cost, physical health, and emotional burden. It should be noted that quality of life is a very subjective term and the measurements in one study may not correlate with the other. Therefore, both options were discussed in length with the patient rather than just with the medical team. This situation stresses the importance of including the patient in the decision-making process and taking into account their specific concerns based on what they deem is an adequate quality of life, regardless of which treatment is more "effective." It is vital for physicians to discuss all treatment options with the patient and provide adequate education about every aspect of the treatment: risk, benefits, and effects on the values the patient identifies as being important to his/her quality of life.

As indicated in other Clinical Decision Reports, using the same outcome questionnaire from clinical research for the ongoing care of the individual patient is a good way to apply the clinical research to clinical practice. For Mr. Smith, the KDQOL-SFTM 1.3 questionnaire can be found at https://www.rand.org/content/dam/rand/pubs/papers/2006/P7994.pdf along with restrictions and use guidelines. 


\section{Conflict Of Interest Statement}

The author declares no conflicts of interest.

\section{References}

1. Jung HY, Jeon Y, Park Y, et al. Better Quality of Life of Peritoneal Dialysis compared to Hemodialysis over a Two-year Period after Dialysis Initiation. Sci Rep. Jul 16 2019;9(1):10266. https://doi.org/10.1038/s41598-019-46744-1

2. Czyżewski L, Sańko-Resmer J, Wyzgał J, Kurowski A. Assessment of health-related quality of life of patients after kidney transplantation in comparison with hemodialysis and peritoneal dialysis. Ann Transplant. Nov 9 2014;19:576-585. https://doi.org/10.12659/AOT.891265

3. Surendra NK, Abdul Manaf MR, Hooi LS, et al. Cost utility analysis of end stage renal disease treatment in Ministry of Health dialysis centres, Malaysia: Hemodialysis versus continuous ambulatory peritoneal dialysis. PLoS One. Oct 23 2019;14(10):e0218422. https://doi.org/10.1371/journal.pone.0218422

4. Majkowicz M, Afeltowicz Z, Lichodziejewska-Niemierko M, Debska-Slizien A, Rutkowski B. Comparison of the quality of life in hemodialysed (HD) and peritoneally dialysed (CAPD) patients using the EORTC QLQ-C30 questionnaire. Int J Artif Organs. 2000;23(7):423-428.

5. Cirillo L, Cutruzzulà R, Somma C, et al. Depressive Symptoms in Dialysis: Prevalence and Relationship with Uremia-Related Biochemical Parameters. Blood Purif. 2018;46(4):286-291. https://doi.org/10.1159/000491014

6. Korevaar JC, Feith GW, Dekkher FW. Effect of starting with hemodialysis compared with peritoneal dialysis in patients new on dialysis treatment: A randomized controlled trial. Kidney International. 2003;64(6):2222-2228. https://doi.org/10.1046/i.15231755.2003.00321.x

7. Ebell MH, Siwek J, Weiss BD, Woolf SH, Susman J, Ewigman B, Bowman M. Strength of recommendation taxonomy (SORT): a patient-centered approach to grading evidence in the medical literature. J Am Board Fam Pract. 2004 Jan-Feb;17(1):59-67. https://doi.org/10.3122/jabfm.17.1.59 